\title{
NUMERICAL STUDY OF NON-NEWTONIAN POLYMERIC BOUNDARY LAYER FLOW AND HEAT TRANSFER FROM A PERMEABLE HORIZONTAL ISOTHERMAL CYLINDER
}

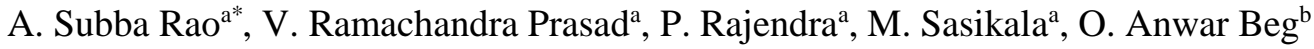 \\ ${ }^{a}$ Department of Mathematics, Madanapalle Institute of Technology and Science, Madanapalle-517325, India. \\ ${ }^{b}$ Fluid Mechanics, Nanosystems and Propulsion, Aeronautical and Mechanical Engineering, School of Computing, Science and Engineering, Newton \\ Building, University of Salford, Manchester M54WT, United Kingdom.
}

\begin{abstract}
In this article, we investigate the nonlinear steady state boundary layer flow and heat transfer of an incompressible Jeffery non-Newtonian fluid from a permeable horizontal isothermal cylinder. The transformed conservation equations are solved numerically subject to physically appropriate boundary conditions using a versatile, implicit, finite-difference technique. The numerical code is validated with previous studies. The influence of a number of emerging non-dimensional parameters, namely with Deborah number (De), surface suction parameter (S), Prandtl number (Pr), ratio of relaxation to retardation times $(\lambda)$ and dimensionless tangential coordinate $(\xi)$ on velocity and temperature evolution in the boundary layer regime are examined in detail. Furthermore, the effects of these parameters on surface heat transfer rate and local skin friction are also investigated. It is found that the velocity is reduced with increasing Deborah number whereas temperature is enhanced. Increasing $\lambda$ enhances the velocity but reduces the temperature. The heat transfer rates is found to be depressed with increasing Deborah number, De, and enhanced with increasing $\lambda$. Local skin friction is found to be decreased with a rise in Deborah number whereas it is elevated with increasing values of relaxation to retardation time ratio ( $\lambda$ ). Increasing suction decelerates the flow and cools the boundary layer i.e. reduces temperatures. With increasing tangential coordinate, the flow is also decelerated whereas the temperatures are enhanced. The simulation is relevant to polymer coating thermal processing. Polymeric enrobing flows are important in industrial manufacturing technology and process systems. Such flows are non-Newtonian. Motivated by such applications, we did the present problem.
\end{abstract}

Keywords: Non-Newtonian fluid; polymers; finite difference numerical method; heat transfer; boundary layers; enrobing flows, skin friction; Deborah number; suction.

\section{INTRODUCTION}

Polymeric flows are generally non-Newtonian in nature. In coating applications, fluid mechanics and heat transfer play a key role in determining the constitution of manufactured polymers Middleman (1997). In dip coating processes for example the surface to be coated is first immersed in a polymer and then steadily withdrawn Roy (1971). Since polymers have high viscosity levels, industrial chemical/manufacturing flow processes exploiting such fluids are generally laminar in nature Baaijens et.al (1997). The rheological nature of polymers also necessitates more sophisticated mathematical models for describing shear stress-strain relationships Otsuki (1999). Important characteristics include relaxation, retardation, viscoelasticity and elongational viscosity. A number of theoretical and computational studies have been communicated on transport phenomena from cylindrical bodies, which frequently arise in polymer processing systems. These Newtonian studies were focused more on heat transfer aspects and include Eswara and Nath Eswara and Nath (1992), Rotte and Beek (1962), and the pioneering analysis of Sakiadis (1961). Further more recent studies examining multi-physical and chemical transport from cylindrical bodies include Zueco et al. (2009, 2011). An early investigation of rheological boundary layer heat transfer from a horizontal cylinder was presented by Chen and Leonard (1972), who considered the power-law model and demonstrated that the transverse curvature has a strong effect on skin friction at moderate and large distances from the leading edge of the boundary layer. Lin and Chen
(1979) also studied axisymmetric laminar boundary-layer convection flow of a power-law non-Newtonian fluid over both a circular cylinder and a spherical body using the Merk-Chao series solution method. Pop et al. (1990) simulated numerically the steady laminar forced convection boundary layer of power-law non-Newtonian fluids on a continuously moving cylinder with the surface maintained at a uniform temperature or uniform heat flux. Further non-Newtonian models employed in analyzing convection flows from cylinders include micropolar liquids chang (2006), viscoelastic materials Anwar et.al (2008) and Kasim et.al (2011), micro polar nanofluids Rehman and Nadeem (2011) and Casson fluids Prasad et.al (2012). One subclass of non-Newtonian fluids known as the Jeffery fluid Saasen and Hassager (1991) is particularly useful owing to its simplicity. This fluid model is capable of describing the characteristics of relaxation and retardation times, which arise in complex polymeric flows. Furthermore, the Jeffrey type model utilizes time derivatives rather than convected derivatives, which make it more amenable for numerical simulations. Recently the Jeffery model has received considerable attention. Interesting studies employing this model include peristaltic magnetohydrodynamic non-Newtonian flow by Kothandapani and Srinivas (2008), MHD Free Convection Flow over an Exponentially Moving Vertical Plate by Srinivasa Raju et al.,(2016), variableviscosity peristaltic flow Nadeem and Akbar (2009), convectiveradiative flow in porous media Hayat et.al (2012) and stretching sheet flows by Hayat and Alsaedi (2012) and Nadeem et.al (2011).

*Corresponding author. Email: subbumaths.a@gamil.com 
The objective of the present paper is to investigate the laminar boundary layer flow and heat transfer of a Jeffery non-Newtonian fluid from a horizontal cylinder. The non-dimensional transport equations with associated dimensionless boundary conditions constitute a highly nonlinear, coupled two-point boundary value problem. Keller's implicit finite difference "box" scheme is implemented to solve the problem. The effects of the emerging thermophysical parameters, namely Prandtl number, Deborah number, ratio of relaxation to retardation times and transpiration (wall suction or injection) on the momentum and heat transfer characteristics are studied. The present problem has to the authors' knowledge not appeared thus far in the scientific literature and is relevant to polymeric manufacturing processes.

\section{MATHEMATICAL MODEL}

Laminar incompressible boundary layer flow and heat transfer under thermal buoyancy force, from a horizontal permeable cylinder to a Jeffery rheological fluid is examined, as illustrated in Figure 1. Both the cylinder and the Jeffery fluid are maintained initially at the same temperature. Instantaneously they are raised to a temperature $T_{w}>T_{\infty}$,

where the latter (ambient) temperature of the fluid is sustained constant. The $\mathrm{x}$-coordinate (tangential) is orientated along the circumference of the horizontal cylinder from the lowest point and the y-coordinate (radial) is directed perpendicular to the surface, with a denoting the radius of the horizontal cylinder. $\Phi=\mathrm{x} / \mathrm{a}$ represents the angle of the $\mathrm{y}-$ axis with respect to the vertical $(0 \leq \Phi \leq \pi)$. The gravitational acceleration $\mathrm{g}$, acts vertically downwards. The Boussineq approximation holds i.e. density variation is only experienced in the buoyancy term in the momentum equation. The Cauchy stress tensor, S, of a Jeffrey nonNewtonian fluid [24] takes the form:

$$
\boldsymbol{T}=-p \boldsymbol{I}+\boldsymbol{S}, \boldsymbol{S}=\frac{\mu}{1+\lambda}\left(\dot{\gamma}+\lambda_{1} \ddot{\gamma}\right)
$$

where a dot above a quantity denotes the material time derivative, $p$ is pressure, $\boldsymbol{I}$ is the identity tensor, $\mu$ is dynamic viscosity, $\lambda$ is the ratio of relaxation to retardation times, $\lambda_{1}$ is the retardation time and $\dot{\gamma}$ is the shear rate. The Jeffery model provides an elegant formulation for simulating retardation and relaxation effects arising in polymer flows. Introducing the boundary layer approximations, and incorporating the stress tensor for a Jeffery fluid in the momentum equation (in differential form) the conservations equations take the form:

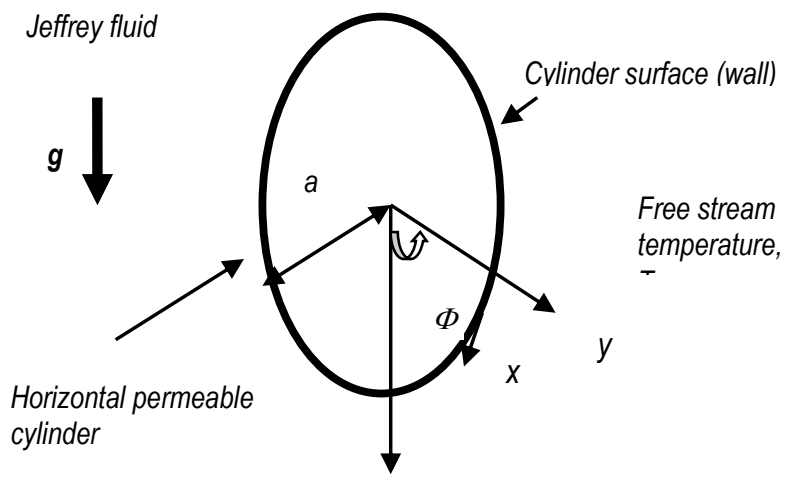

Fig. 1 Physical model and coordinate system

$$
\begin{aligned}
& \frac{\partial u}{\partial x}+\frac{\partial v}{\partial y}=0 \\
& u \frac{\partial u}{\partial x}+v \frac{\partial u}{\partial y}=g \beta\left(T-T_{\infty}\right) \sin \left(\frac{x}{a}\right) \\
& +\frac{v}{1+\lambda}\left(\frac{\partial^{2} u}{\partial y^{2}}+\lambda 1\left(u \frac{\partial^{3} u}{\partial x \partial y^{2}}-\frac{\partial u}{\partial x} \frac{\partial^{2} u}{\partial y^{2}}+\frac{\partial u}{\partial y} \frac{\partial^{2} u}{\partial x \partial y}+v \frac{\partial^{3} u}{\partial y^{3}}\right)\right)
\end{aligned}
$$

$$
u \frac{\partial T}{\partial x}+v \frac{\partial T}{\partial y}=\frac{k}{\rho c_{p}} \frac{\partial^{2} T}{\partial y^{2}}
$$

The Jeffery fluid model therefore introduces a number of mixed derivatives into the momentum boundary layer equation (3) and in particular two third order derivatives, making the system an order higher than the classical Navier-Stokes (Newtonian) viscous flow model. The non-Newtonian effects feature in the shear terms only of eqn. (3) and not the convective (acceleration) terms. The final term on the right hand side of eqn. (3) represents the thermal buoyancy effect (free convection) and couples eqn. (3) to the energy equation (4). Viscous dissipation effects are neglected in the model. In eqns. (2)-(4) $u$ and $v$ designate velocity components in the $x$ - and $y$-directions respectively, $v=\frac{\mu}{\rho}$ is the kinematic viscosity of the Jeffery fluid, $\beta$ is the coefficient of thermal expansion, $\alpha$ is the thermal diffusivity, $T$ is the temperature, $k$ is the thermal conductivity of the Jeffery fluid, $\rho$ is the density of the Jeffery fluid, $c_{p}$ is the specific heat at constant pressure, and all other parameters have been defined earlier. The appropriate boundary conditions are imposed at the cylinder surface and in the free stream (edge of the boundary layer) and take the form:

At $y=0, \quad u=0, v=-V_{w}, \quad T=T_{w}$

As $y \rightarrow \infty, \quad u \rightarrow 0, \quad v \rightarrow 0, \quad T \rightarrow T_{\infty}$

$V_{w}$ denotes the uniform transpiration (blowing or suction) velocity at the surface of the permeable cylinder. To transform the boundary value problem to a dimensionless one, we introduce a stream function $\psi$ defined by the Cauchy-Riemann equations, $u=\frac{\partial \psi}{\partial y}$ and $v=-\frac{\partial \psi}{\partial x}$, and therefore, the mass conservation eqn. (2) is automatically satisfied. Furthermore, the following dimensionless variables are introduced into eqns. (2)-(4) and (5):

$$
\begin{array}{ll}
\xi=\frac{x}{a}, \quad \eta=\frac{y}{a} \sqrt[4]{G r}, \quad f(\xi, \eta)=\frac{\psi}{v \xi \sqrt[4]{G r}}, \quad \operatorname{Pr}=\frac{k}{\rho c_{p}} \\
S=-\frac{V_{w} a}{v^{4} \sqrt{G r}}, G r=\frac{g \beta\left(T_{w}-T_{\infty}\right) a^{3}}{v^{2}}, \quad D e=\frac{\lambda_{1} v \sqrt{G r}}{a^{2}}
\end{array}
$$

Where $\xi$ is the non-dimensional tangential coordinate, $\eta$ is the nondimensional radial coordinate, $f$ is dimensionless stream function, $\operatorname{Pr}$ is the Prandtl number, $S$ is the wall transpiration parameter $(S>0$ for suction and $S<0$ for injection, the case of an impermeable cylinder is retrieved for $S=0$ ), $G r$ is the Grashof number and $D e$ is the Deborah number characterizing the fluidity of the material (viscoelasticity). The resulting momentum and thermal boundary layer equations take the form:

$$
\begin{aligned}
& \frac{1}{1+\lambda} f^{\prime \prime \prime}+\frac{D e}{1+\lambda}\left[f^{\prime \prime 2}-f f^{i v}\right]+f f^{\prime \prime}-f^{\prime 2}+\frac{\sin \xi}{\xi} \theta= \\
& \xi\left(f^{\prime} \frac{\partial f^{\prime}}{\partial \xi}-f^{\prime \prime} \frac{\partial f}{\partial \xi} \frac{D e}{1+\lambda}\left[f^{\prime} \frac{\partial f^{\prime \prime \prime}}{\partial \xi}-f^{\prime \prime \prime} \frac{\partial f^{\prime}}{\partial \xi}+f^{\prime \prime} \frac{\partial f^{\prime \prime}}{\partial \xi}-f^{i v} \frac{\partial f}{\partial \xi}\right]\right) \\
& \frac{\theta^{\prime \prime}}{\operatorname{Pr}}+f \theta^{\prime}=\frac{7}{4} \xi\left(f^{\prime} \frac{\partial \theta}{\partial \xi}-\theta^{\prime} \frac{\partial f}{\partial \xi}\right)
\end{aligned}
$$

The location, $\xi \sim 0$, corresponds to the vicinity of the lower stagnation point on the cylinder, an aspect discussed in more detail subsequently. The corresponding non-dimensional boundary conditions for the collectively sixth order, multi-degree partial differential equation system defined by eqns. (7), (8) assume the form:

$$
\begin{aligned}
& \text { At } \eta=0, \quad f=S, \quad f^{\prime}=0, \quad \theta=1 \\
& \text { As } \eta \rightarrow \infty, \quad f^{\prime} \rightarrow 0, \quad f^{\prime \prime} \rightarrow 0, \quad \theta \rightarrow 0
\end{aligned}
$$


Here primes denote the differentiation with respect to $\eta$. The skinfriction coefficient (shear stress at the cylinder surface) and Nusselt number (heat transfer rate at the cylinder surface) can be defined using the transformations described above with the following expressions:

$$
\begin{aligned}
& \frac{1}{2} G r^{-3 / 4} C_{f}=f^{\prime \prime}(\xi, 0) \\
& G r^{-1 / 4} N u=-\theta^{\prime}(\xi, 0)
\end{aligned}
$$

\section{COMPUTATIONAL SOLUTION WITH KELLER BOX IMPLICT METHOD}

In Purely analytical solutions for the boundary value problem defined by eqns. (7), (8) and conditions (10) are extremely difficult, if not intractable. A computational solution is therefore developed using a versatile and stable finite difference algorithm based on Keller's box method Keller (1970). This implicit method has been implemented extensively in non-Newtonian fluid mechanics simulations for a variety of different rheological models. Hossain et al. (1970) used a power-law model and Keller's scheme to study free convection boundary layers from a slotted vertical plate. Javed et al. (2013) employed the EyringPowell rheological model and the Keller box method to simulate stretching sheet boundary layer flow. Beg et al. (2001) used Keller's box algorithm to study magneto-viscoelastic natural convection from a wedge in porous media. Further nonlinear thermal convection studies using Keller's box method include Vajravelu et al. (2012), Bég et al. (2011). The present code has received extensive validation in previous studies, as described in Subbarao et.al (2016) and therefore confidence is high in the accuracy of computations.

The fundamental steps of the Keller Box Scheme are as follows:

1) Reduction of the $N^{\text {th }}$ order partial differential equation system to $N$ first order equations

2) Finite Difference Discretization

3) Quasilinearization of Non-Linear Keller Algebraic Equations

4) Block-tridiagonal Elimination of Linear Keller Algebraic Equations.

\section{Step 1: Reduction of the $N^{\text {th }}$ order partial differential equation} system to $N$ first order equations

New variables are introduced to Eqns. (7) - (8) and (9), to render the boundary value problem as a multiple system of first order equations. A set of six simultaneous first order differential equations are therefore generated by introducing the new variables $u, v, q$ and $t$ :

$$
\begin{aligned}
& f^{\prime}=u \\
& u^{\prime}=v \\
& v^{\prime}=q \\
& \theta^{\prime}=t \\
& \frac{v^{\prime}}{1+\lambda}+\frac{D e}{1+\lambda}\left(v^{2}-f q^{\prime}\right)+f v-u^{2}+\frac{\sin \xi}{\xi} s \\
& =\left(u \frac{\partial u}{\partial \xi}-v \frac{\partial f}{\partial \xi}-\frac{D e}{1+\lambda}\left(u \frac{\partial q}{\partial \xi}-q \frac{\partial u}{\partial \xi}+v \frac{\partial v}{\partial \xi}-q^{\prime} \frac{\partial f}{\partial \xi}\right)\right) \\
& \frac{1}{\operatorname{Pr}} t^{\prime}+f t=\xi\left(u \frac{\partial s}{\partial \xi}-t \frac{\partial f}{\partial \xi}\right)
\end{aligned}
$$

where primes denote differentiation with respect to $\eta$. In terms of the dependent variables, the boundary conditions become:

At

$$
\eta=0: \quad u=0, \quad f=S, \quad \theta=1
$$

As

$$
\eta \rightarrow \infty: \quad u \rightarrow 0, \quad v \rightarrow 0, \quad \theta \rightarrow 0
$$

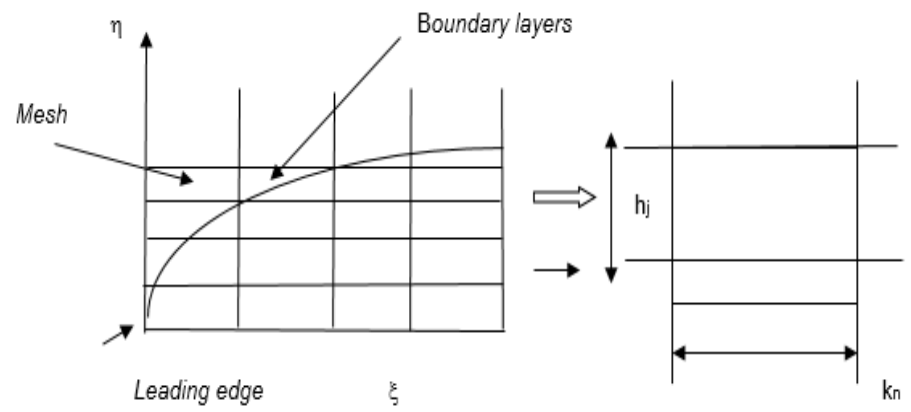

Fig.2 Keller Box element and boundary layer mesh

\section{Step 2: Finite Difference Discretization}

A two dimensional computational grid is imposed on the $\xi-\eta$ plane as sketched in Fig. 2. The stepping process is defined by:

$\eta_{0}=0, \quad \eta_{j}=\eta_{j-1}+h_{j}, j=1,2, \ldots \ldots J, \eta_{j}=\eta_{\infty}$

$\xi^{0}=0, \quad \xi^{n}=\xi^{n-1}+k_{n}, \quad n=1,2, \ldots . N$

Where $k_{n}$ and $h_{j}$ denote the step distances in the $\xi$ and $\eta$ directions respectively.

If $g_{j}^{n}$ denotes the value of any variable at $\left(\eta_{j}, \xi^{n}\right)$, then the variables and derivatives of Eqns. $(12 \mathrm{a})-(12 \mathrm{f})$ at $\left(\eta_{j-1 / 2}, \xi^{n-1 / 2}\right)$ are replaced by:

$$
\begin{aligned}
& g_{j-1 / 2}^{n-1 / 2}=\frac{1}{4}\left(g_{j}^{n}+g_{j-1}^{n}+g_{j}^{n-1}+g_{j-1}^{n-1}\right) \\
& \left(\frac{\partial g}{\partial \eta}\right)_{j-1 / 2}^{n-1 / 2}=\frac{1}{2 h_{j}}\left(g_{j}^{n}-g_{j-1}^{n}+g_{j}^{n-1}-g_{j-1}^{n-1}\right) \\
& \left(\frac{\partial g}{\partial \xi}\right)_{j-1 / 2}^{n-1 / 2}=\frac{1}{2 k^{n}}\left(g_{j}^{n}-g_{j-1}^{n}+g_{j}^{n-1}-g_{j-1}^{n-1}\right)
\end{aligned}
$$

The resulting finite-difference approximation of equations (12a) - (12f) for the mid-point $\left(\eta_{j-1 / 2}, \xi^{n}\right)$, take the form:

$$
\begin{aligned}
& h_{j}^{-1}\left(f_{j}^{n}-f_{j-1}^{n}\right)=u_{j-1 / 2}^{n} \\
& h_{j}^{-1}\left(u_{j}^{n}-u_{j-1}^{n}\right)=v_{j-1 / 2}^{n} \\
& h_{j}^{-1}\left(v_{j}^{n}-v_{j-1}^{n}\right)=q_{j-1 / 2}^{n} \\
& h_{j}^{-1}\left(\theta_{j}^{n}-\theta_{j-1}^{n}\right)=t_{j-1 / 2}^{n} \\
& \frac{\left(v_{j}-v_{j-1}\right)}{1+\lambda}+\frac{D e}{1+\lambda} \frac{(1+\alpha) h_{j}}{4}\left(v_{j}+v_{j-1}\right)^{2}-\frac{D e}{1+\lambda} \frac{(1+\alpha)}{2} \\
& {\left[\left(f_{j}+f_{j-1}\right)\left(q_{j}-q_{j-1}\right)\right]+\frac{(1+\alpha) h_{j}}{4}\left[\left(f_{j}+f_{j-1}\right)\left(v_{j}+v_{j-1}\right)\right]-} \\
& (1+\alpha) \frac{h_{j}}{4}\left(u_{j}+u_{j-1}\right)^{2}+\frac{B h_{j}}{2}\left(s_{j}+s_{j-1}\right)+\frac{\alpha h_{j}}{2} v_{j-1 / 2}^{n-1}\left(f_{j}+f_{j-1}\right) \\
& -\frac{\alpha h_{j}}{2} f_{j-1 / 2}^{n-1}\left(v_{j}+v_{j-1}\right)-\alpha h_{j} \frac{D e}{1+\lambda} q_{j-1}^{n-1}\left(u_{j}+u_{j-1}\right)+ \\
& \alpha h_{j} \frac{D e}{1+\lambda} u_{j-1}^{n-1}\left(q_{j}+q_{j-1}\right)+\frac{\alpha h_{j}}{2} \frac{D e}{1+\lambda} q_{j-1}^{n-1}\left(u_{j}+u_{j-1}\right)+ \\
& \alpha h_{j} \frac{D e}{1+\lambda} u_{j-1}^{n-1}\left(q_{j}+q_{j-1}\right)+\frac{\alpha h_{j}}{2} \frac{D e}{1+\lambda} f_{j-1}^{n-1}\left(q_{j}-q_{j-1}\right) \\
& -\frac{\alpha h_{j}}{2} \frac{D e}{1+\lambda}\left(q^{\prime}\right)_{j-1}^{n-1}\left(f_{j}+f_{j-1}\right)=\left[R_{1}\right]_{j-1 / 2}^{n-1}
\end{aligned}
$$




$$
\begin{aligned}
& \frac{1}{\operatorname{Pr}}\left(t_{j}-t_{j-1}\right)+\frac{(1+\alpha) h_{j}}{4}\left[\left(f_{j}+f_{j-1}\right)\left(t_{j}+t_{j-1}\right)\right] \\
& -\frac{\alpha h_{j}}{4}\left[\left(u_{j}+u_{j-1}\right)\left(s_{j}+s_{j-1}\right)\right]+\frac{\alpha h_{j}}{2} s_{j-1 / 2}^{n-1}\left(u_{j}+u_{j-1}\right) \\
& -\frac{\alpha h_{j}}{2} u_{j-1 / 2}^{n-1}\left(s_{j}+s_{j-1}\right)-\frac{\alpha h_{j}}{2} f_{j-1 / 2}^{n-1}\left(t_{j}+t_{j-1}\right) \\
& +\frac{\alpha h_{j}}{2} t_{j-1 / 2}^{n-1}\left(f_{j}+f_{j-1}\right)=\left[R_{2}\right]_{j-1 / 2}^{n-1}
\end{aligned}
$$

Where the following notation applies

$$
\begin{aligned}
& \alpha=\frac{\xi^{n-1 / 2}}{k_{n}} \quad B=\frac{\sin \left(\xi^{n-1 / 2}\right)}{\xi^{n-1 / 2}} \\
& {\left[R_{1}\right]_{j-1 / 2}^{n-1}=-h_{j}\left[\begin{array}{c}
\frac{1}{1+\lambda}\left(v^{\prime}\right)_{j-1 / 2}^{n-1}+(1-\alpha) f_{j-1 / 2}^{n-1} v_{j-1 / 2}^{n-1}+\frac{D e}{1+\lambda}(1-\alpha)\left(v_{j-1}^{n-1}\right)^{2} \\
-\frac{D e}{1+\lambda}(1-\alpha) f_{j-1 / 2}^{n-1}\left(q^{\prime}\right)_{j-1 / 2}^{n-1}+B s_{j-1 / 2}^{n-1}-(1-\alpha)\left(u_{j-1}^{n-1}\right)^{2}
\end{array}\right]} \\
& {\left[R_{2}\right]_{j-1 / 2}^{n-1}=-h_{j}\left[\frac{1}{\operatorname{Pr}}\left(\frac{t_{j}-t_{j-1}}{h_{j}}\right)+(1-\alpha)\left(f_{j-1 / 2} t_{j-1 / 2}\right)+\alpha\left(u_{j-1 / 2} s_{j-1 / 2}\right)\right](1}
\end{aligned}
$$

The boundary conditions are

$f_{0}^{n}=u_{0}^{n}=0, \theta_{0}^{n}=1, \quad u_{j}^{n}=0, \quad v_{j}^{n}=0, \quad \theta_{j}^{n}=0$

Step 3: Quasilinearization of Non-Linear Keller Algebraic Equations Assuming $f_{j-1}^{n-1}, u_{j-1}^{n-1}, v_{j-1}^{n-1}, q_{j-1}^{n-1}, s_{j-1}^{n-1}, t_{j-1}^{n-1}$, to be known for $0 \leq j \leq J$, then Eqns. (16a) - (16f) constitute a system of $6 J+6$ equations for the solution of $6 \mathrm{~J}+6$ unknowns $f_{j}^{n}, u_{j}^{n}, v_{j}^{n}, q_{j}^{n}, s_{j}^{n}, t_{j}^{n}, j=0,1,2 \ldots, J$. This nonlinear system of algebraic equations is linearized by means of Newton's method as explained in Subba Rao et.al. $(2015,2016,2017)$.

\section{Step 4: Block-tridiagonal Elimination of Linear Keller Algebraic Equations}

The linear system (15a) - (15f) can now be solved by the blockelimination method owing to its block-tridiagonal structure. The bocktridiagonal structure generated consists of block matrices. The complete linearized system is formulated as a block matrix system, where each element in the coefficient matrix is a matrix itself, and this system is solved using the efficient Keller-box method. The numerical results are strongly influenced by the number of mesh points in both directions. After some trials in the $\eta$-direction (radial coordinate), a larger number of mesh points are selected whereas in the $\xi$-direction (tangential coordinate) significantly less mesh points are necessary. $\eta_{\max }$ has been set at 10 and this constitutes an adequately large value at which the prescribed boundary conditions are satisfied. $\xi_{\max }$ is set at 3.0 for the simulations. Mesh independence has been comfortably attained in the simulations. The numerical algorithm is executed in MATLAB.

\section{RESULTS AND DISCUSSION}

Comprehensive solutions have been obtained and are presented in Tables 1 and 2 and Figs. 3-7. Table 1 presents the influence of increasing Prandtl number on skin friction and heat transfer rate, along with a variation in the parameter $(\lambda)$, ratio of relaxation and retardation times and transverse coordinate $(\xi)$. With increasing Prandtl number, the skin friction is generally decreased, whereas heat transfer rate is markedly enhanced. Heat transfer rate is maximized at the lower stagnation point $(\xi=0)$, for any value of Prandtl number or rheological parameter $(\lambda)$. With an increase in $\lambda$, both skin friction and heat transfer rates are increased. This implies that as the relaxation time is reduced (and the retardation time increased) the polymer flows faster and transfers heat more efficiently from the cylinder surface. This appears consistent with other studies Hayat et.al. (2012). in table 1, $S$ is positive corresponding to wall suction.

Table: 1 Values of $f^{\prime \prime}(\xi, 0)$ and $-\theta^{\prime}(\xi, 0)$ and different values of $\lambda, \operatorname{Pr}$ and $\xi(\mathrm{De}=0.1, \mathrm{~S}=0.5)$

\begin{tabular}{|c|c|c|c|c|c|c|}
\hline \multirow{4}{*}{$\wedge$} & $\operatorname{Pr}$ & $\xi=0.0$ & \multicolumn{2}{|c|}{$\xi=0.5$} & \multicolumn{2}{|c|}{$\xi=1.0$} \\
\cline { 3 - 7 } & & $-\theta^{\prime}(\xi, 0)$ & $f^{\prime \prime}(\xi, 0)$ & $-\theta^{\prime}(\xi, 0)$ & $f^{\prime \prime}(\xi, 0)$ & $-\theta^{\prime}(\xi, 0)$ \\
\hline \multirow{5}{*}{0.0} & 0.5 & 0.7560 & 0.4233 & 0.4159 & 0.8145 & 0.4960 \\
\cline { 2 - 7 } & 0.7 & 0.9422 & 0.3931 & 0.4901 & 0.7480 & 0.6116 \\
\cline { 2 - 7 } & 1.0 & 1.2142 & 0.3564 & 0.5890 & 0.6671 & 0.7843 \\
\cline { 2 - 7 } & 2.0 & 2.1272 & 0.2749 & 0.8379 & 0.4875 & 1.3844 \\
\cline { 2 - 7 } & 5.0 & 5.0300 & 0.1601 & 1.1561 & 0.2420 & 3.6022 \\
\hline & 7.0 & 7.0147 & 0.1236 & 1.1563 & 0.1664 & 5.3749 \\
\hline \multirow{5}{*}{0.5} & 0.5 & 0.7774 & 0.5696 & 0.4315 & 1.1081 & 0.5090 \\
\cline { 2 - 7 } & 0.7 & 0.9672 & 0.5313 & 0.5113 & 1.0228 & 0.6272 \\
\cline { 2 - 7 } & 1.0 & 1.2422 & 0.4846 & 0.6169 & 0.9186 & 0.8018 \\
\cline { 2 - 7 } & 2.0 & 2.1541 & 0.3798 & 0.8884 & 0.6851 & 1.3966 \\
\cline { 2 - 7 } & 5.0 & 5.0405 & 0.2277 & 1.2787 & 0.3564 & 3.5334 \\
\cline { 2 - 7 } & 7.0 & 7.0205 & 0.1770 & 1.3203 & 0.2505 & 5.2395 \\
\hline
\end{tabular}

Table 2 presents the influence of increasing Deborah number parameter, Deon skin friction and heat transfer rate, along with a variation in the suction parameter, $S$ and transverse coordinate, $\xi$. With increasing Deborah number, the skin friction is generally decreased, and heat transfer rate is also decreased. This trend is sustained for all values of transverse coordinate. An increase in suction $(S>0)$ reduces skin friction at higher values of the transverse coordinate, for any value of Deborah number. Further from the vicinity of the lower stagnation point, therefore the polymer flow is decelerated with suction. However, at lower values of transverse coordinate, suction slightly accelerates the flow for all Deborah numbers. In the vicinity of the lower stagnation point, $\xi \rightarrow 0$ and the boundary layer equations eqns. (7) to (8) contract to a system of ordinary differential equations:

Table: 2 Values of $f^{\prime \prime}(\xi, 0)$ and $-\theta^{\prime}(\xi, 0)$ and different values of $\mathrm{S}$, De and $\xi(\lambda=0.2, \operatorname{Pr}=0.71)$

\begin{tabular}{|c|c|c|c|c|c|c|}
\hline \multirow{3}{*}{$S$} & \multirow{2}{*}{ De } & $\xi=0.0$ & \multicolumn{2}{|c|}{$\xi=0.5$} & \multicolumn{2}{|c|}{$\xi=1.0$} \\
\cline { 3 - 7 } & & $-\theta^{\prime}(\xi, 0)$ & $f^{\prime \prime}(\xi, 0)$ & $-\theta^{\prime}(\xi, 0)$ & $f^{\prime \prime}(\xi, 0)$ & $-\theta^{\prime}(\xi, 0)$ \\
\hline \multirow{5}{*}{0.1} & 0.1 & 0.6323 & 0.3846 & 0.3947 & 0.8681 & 0.4158 \\
\cline { 2 - 7 } & 0.2 & 0.6269 & 0.3826 & 0.3947 & 0.8632 & 0.4134 \\
\cline { 2 - 7 } & 0.3 & 0.6217 & 0.3798 & 0.3926 & 0.8585 & 0.4113 \\
\cline { 2 - 7 } & 0.4 & 0.6166 & 0.3769 & 0.3913 & 0.8541 & 0.4092 \\
\cline { 2 - 7 } & 0.5 & 0.6118 & 0.3739 & 0.3899 & 0.8496 & 0.4072 \\
\hline \multirow{5}{*}{0.5} & 0.1 & 0.9629 & 0.4488 & 0.5035 & 0.8583 & 0.6247 \\
\cline { 2 - 7 } & 0.2 & 0.9481 & 0.4397 & 0.5028 & 0.8005 & 0.6247 \\
\cline { 2 - 7 } & 0.3 & 0.9365 & 0.4249 & 0.5000 & 0.7570 & 0.6172 \\
\cline { 2 - 7 } & 0.4 & 0.9269 & 0.4091 & 0.4967 & 0.7206 & 0.6118 \\
\cline { 2 - 7 } & 0.5 & 0.9187 & 0.3938 & 0.4933 & 0.6890 & 0.6076 \\
\hline
\end{tabular}

$\left(\frac{1}{1+\lambda}\right) f^{\prime \prime \prime}+\frac{D e}{1+\lambda}\left(f^{\prime \prime 2}-f f^{i v}\right)+f f^{\prime \prime}-f^{\prime 2}+\theta=0$

$\frac{\theta^{\prime \prime}}{\operatorname{Pr}}+f \theta^{\prime}=0$ 
since $\frac{\sin \xi}{\xi} \rightarrow 0 / 0$ i.e. 1 , so that $\frac{\sin \xi}{\xi} \theta \rightarrow \theta$. At the upper stagnation point, $\xi \sim \pi$.

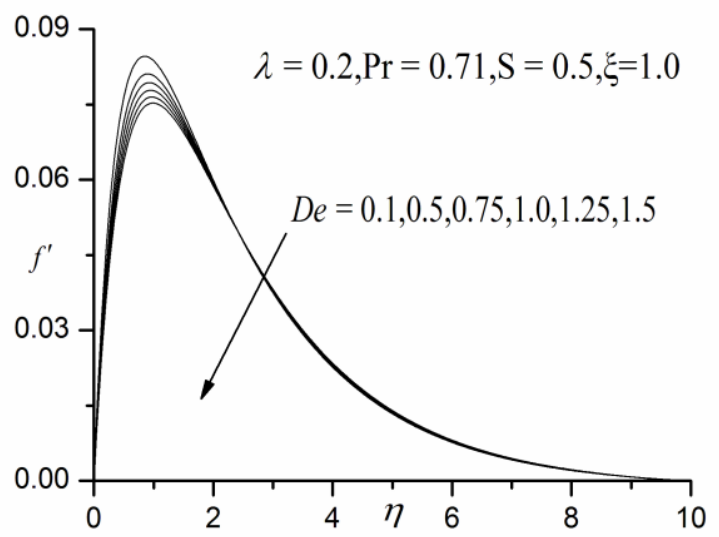

(a)

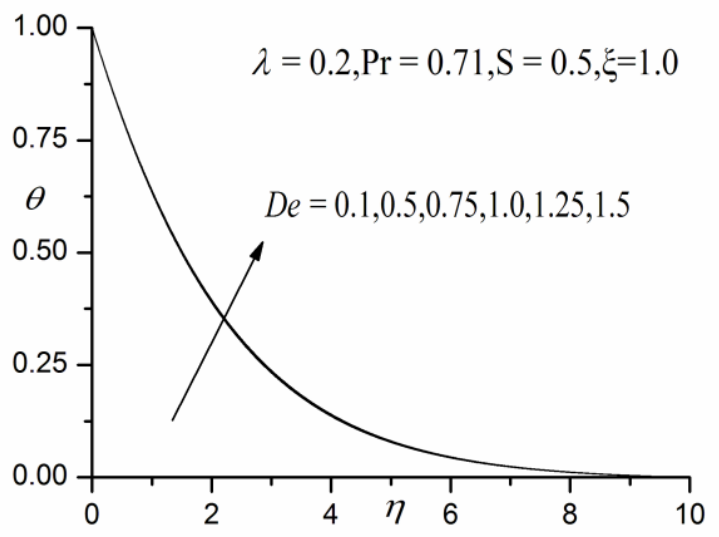

(b)

Fig. 3 Effect of $D e$ on (a) velocity profiles (b) temperature profiles

In Figures 3(a)-3(b), the evolution of velocity and temperature functions with a variation in Deborah number, $D e$, is depicted. Dimensionless velocity component (fig. 3a) is considerably reduced with increasing $D e$ near the cylinder surface and for some distance into the boundary layer. De clearly arises in connection with some high order derivatives in the momentum boundary layer equation, (7) i.e. $\frac{D e}{1+\lambda}\left[f^{\prime \prime 2}-f f^{i v}\right]$ and also $\xi\left(-\frac{D e}{1+\lambda}\left[f^{\prime} \frac{\partial f^{\prime \prime \prime}}{\partial \xi}-f^{\prime \prime \prime} \frac{\partial f^{\prime}}{\partial \xi}+f^{\prime \prime} \frac{\partial f^{\prime \prime}}{\partial \xi}-f^{i v} \frac{\partial f}{\partial \xi}\right]\right)$. It therefore is intimately associated with the shearing characteristics of the polymer flow. For polymers, larger $D e$ values imply that the polymer becomes highly oriented in one direction and stretched. Generally, this arises when the polymer takes longer to relax in comparison with the rate at which the flow is deforming it. When such fluids are stretched, there is a delay in their return to the unstressed state. For very large Deborah numbers, the fluid movement is too fast for elastic forces to relax and the material then acts like a purely elastic solid. Large Deborah numbers are therefore not relevant to the present simulations. For small Deborah numbers, the time scale of fluid movement is much greater than the relaxation time of elastic forces in the polymer and the polymer then behaves as a simple viscous fluid, as elaborated by Bég and Makinde (2011). Vrentas et al. (1975) have also indicated that the Deborah number can be utilized in characterizing diffusional transport in amorphous polymer-solvent systems. Further from the cylinder surface, we observe that there is a slight increase in velocity i.e. the flow is accelerated with increasing Deborah number. With greater distance from the solid boundary, the polymer is therefore assisted in flowing even with higher elastic effects. Clearly, the responses in the near-wall region and far-field region are very different. In fig. $3 b$, an increase in Deborah number is seen to considerably enhance temperatures throughout the boundary layer regime. This has also been observed by Hayat et al. (2012). Although De does not arise in the thermal boundary layer equation (8), there is a strong coupling of this equation with the momentum field via the convective terms $\xi\left[f^{\prime} \frac{\partial \theta}{\partial \xi}\right]$ and $\xi\left[-\theta^{\prime} \frac{\partial f}{\partial \xi}\right]$. Furthermore the thermal buoyancy force term, $+\frac{\sin \xi}{\xi} \theta$, in the momentum equation (7) strongly couples the momentum flow field to the temperature field. With greater elastic effects, it is anticipated that thermal conduction plays a greater role in heat transfer in the polymer. Thermal boundary layer thickness is also elevated with increasing Deborah number.

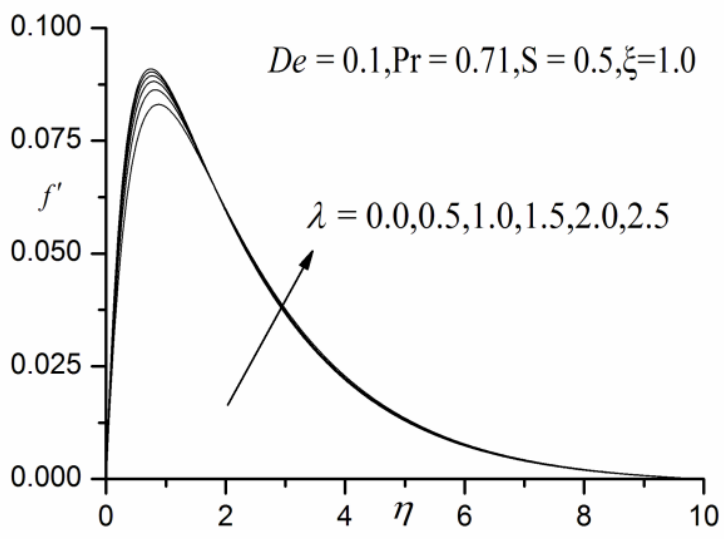

(a)

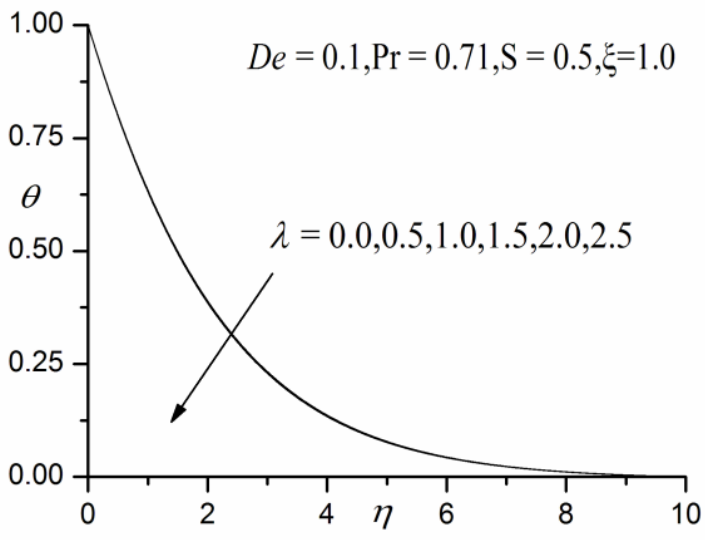

(b)

Fig.4 Effect of $\lambda$ on (a) velocity profiles (b) temperature profiles

Figures 4(a) - 4(b) illustrates the effect of the ratio of relaxation to retardation times i.e. $\lambda$ on the velocity $\left(f^{\prime}\right)$ and temperature distributions through the boundary layer regime. Velocity is significantly decreased with increasing $\lambda$, in particular close to the cylinder surface. The polymer flow is therefore considerably decelerated with an increase in relaxation time (or decrease in retardation time). Conversely, temperature is depressed slightly with 
increasing values of $\lambda$. The mathematical model reduces to the Newtonian viscous flow model as $\lambda \rightarrow 0$ and $D e \rightarrow 0$, since this negates relaxation, retardation and elasticity effects. The momentum boundary layer equation in this cases contracts to the familiar equation for Newtonian mixed convection from a cylinder:

$$
f^{\prime \prime \prime}+f f^{\prime \prime}-f^{\prime 2}+\frac{\sin \xi}{\xi} \theta=\xi\left(f^{\prime} \frac{\partial f^{\prime}}{\partial \xi}-f^{\prime \prime} \frac{\partial f}{\partial \xi}\right)
$$

The thermal boundary layer equation (8) remains unchanged. Effectively with greater relaxation time of the polymer, the thermal boundary layer thickness is reduced. However, with greater relaxation times, the momentum boundary layer thickness is only decreased near the cylinder surface whereas further away it is enhanced since the flow is strongly accelerated there.

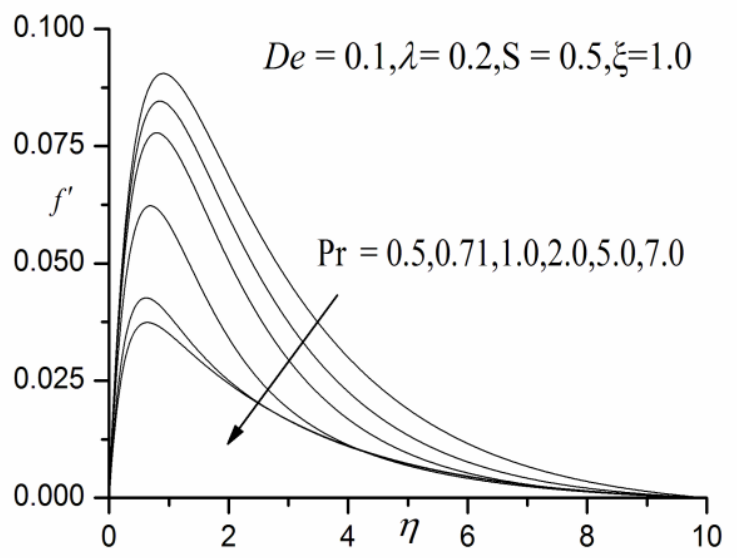

(a)

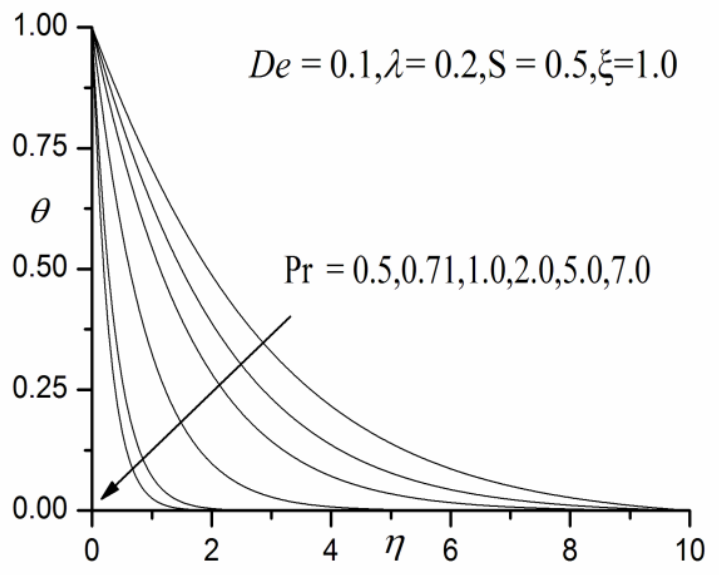

(b)

Fig. 5 Effect of $\operatorname{Pr}$ on (a) velocity profiles (b) temperature

Figures 5(a)-5(b) present typical profiles for velocity $\left(f^{\prime}\right)$ and temperature for various values of Prandtl number, $P r$. It is observed that an increase in the Prandtl number $\operatorname{Pr}$ massively reduces the velocity i.e. decelerates the polymeric boundary layer flow. With increasing Prandtl number, the dynamic viscosity of the fluid is strongly elevated and this is representative of non-Newtonian polymers. The flow is therefore retarded and momentum boundary layer thickness is decreased. At high Prandtl number, thermal conduction heat transfer dominates over thermal convection heat transfer and thermal boundary layer thickness is decreased. Conversely, for lower Prandtl numbers the opposite behavior is observed. Effectively a rise in Prandtl number decreases fluid temperatures and cools the regime.

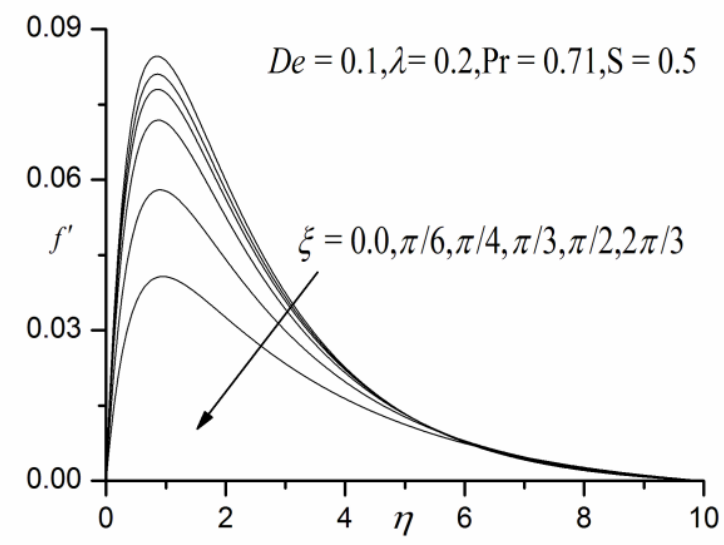

(a)

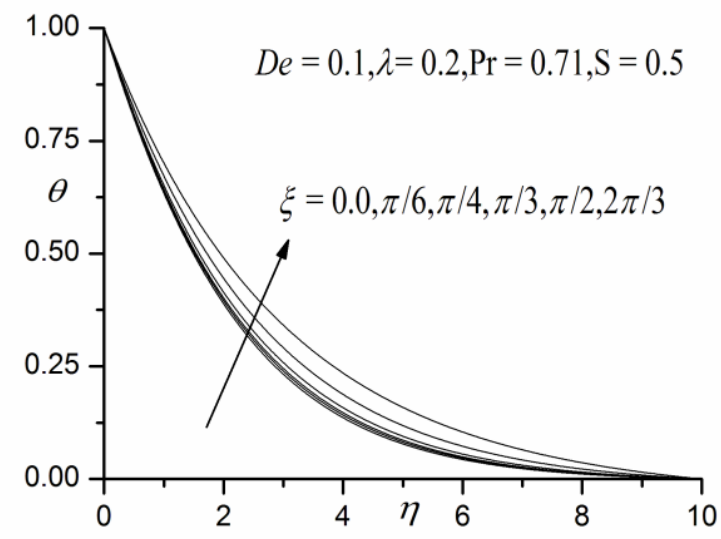

(b)

Fig. 6 Effect of $\xi$ on (a) velocity profiles (b) temperature profiles

Figures 6(a) - 6(b) depict the velocity and temperature distributions with radial coordinate, for various transverse (stream wise) coordinate values, $\xi$. Generally, velocity is noticeably lowered with increasing migration from the leading edge i.e. larger $\xi$ values (figure $6 a)$. The maximum velocity is computed at the lower stagnation point $(\xi \sim 0)$ for low values of radial coordinate $(\eta)$. The transverse coordinate clearly exerts a significant influence on momentum development. A very strong increase in temperature $(\theta)$, as observed in figure $6 \mathrm{~b}$, is generated throughout the boundary layer with increasing $\xi$ values. The temperature field decays monotonically. Temperature is maximized at the cylinder surface $(\eta=0)$ and minimized in the free stream $(\eta=8)$. Although the behavior at the upper stagnation point $(\xi \sim \pi)$ is not computed, the pattern in figure $6 \mathrm{~b}$ suggests that temperature will continue to progressively grow here compared with previous locations on the cylinder surface (lower values of $\xi$ ). Similar observations have been recorded by a number of researchers including Chen and Leonard (1972) for power-law fluids, Chang (2006) for micro polar fluids and Anwar et al. (2008) for viscoelastic fluids.

Figures 7(a) - 7(b) present typical profiles for velocity $\left(f^{\prime}\right)$ and temperature for various values of the transpiration parameter, $S$. As in all other graphs, only the case of wall suction is studied $(S>0)$. It is observed that an increase in the suction parameter significantly decelerates the flow for all values of radial coordinate. The boundary layer thickness is reduced and suction causes the boundary layer to 
adhere closer to the wall. Similarly increasing wall suction is found to lower temperatures in the boundary layer regime and strongly decreases thermal boundary layer thickness. Although boundary layer separation has not been identified in the present regime, suction has been shown to delay this effect in certain viscoelastic cylinder flow problems.

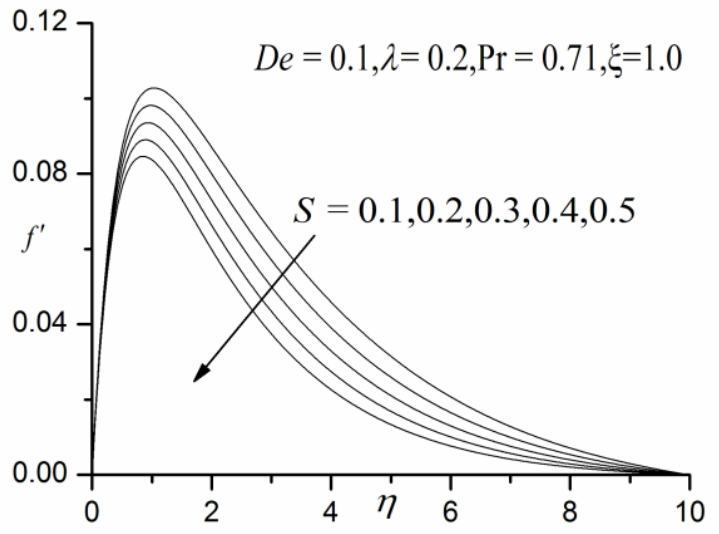

(a)

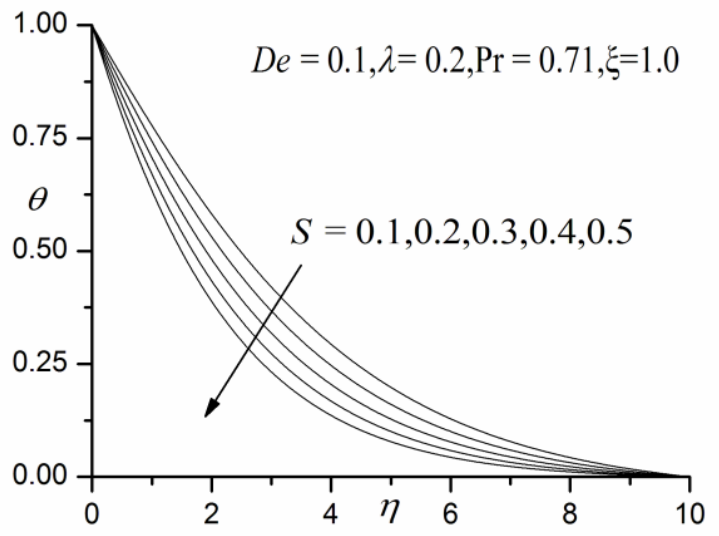

(b)

Fig. 7 Effect of $S$ on (a) velocity profiles (b) temperature profiles

\section{CONCLUSIONS}

A mathematical model has been developed for boundary layer mixed convection flow of a Jeffery non-Newtonian fluid from a horizontal cylinder, with wall transpiration. The transformed conservation equations have been solved with prescribed boundary conditions using the implicit Keller-box finite difference method. The present simulations have shown that:

1. Increasing the viscoelasticity parameter i.e. Deborah number $(D e)$, reduces the velocity, skin friction and heat transfer rate whereas it enhances temperature.

2 . Increasing the parameter ratio of relaxation and retardation times $(\lambda)$, increases velocity, skin friction coefficient and heat transfer rate whereas it reduces temperature.

3. Increasing Prandtl number $(P r)$ markedly reduces velocity and temperature.

4. Increasing transverse coordinate ( $\xi$ generally decelerates the flow near the cylinder surface and reduces momentum boundary layer

thickness whereas it enhances temperature. Heat transfer rate is also maximized at the lower stagnation point $(\xi=0)$.

5. Increasing suction at the cylinder surface $\left(f_{w}>0\right)$ decelerates the flow and strongly depresses temperature.

Generally, very stable and accurate solutions are obtained with the present finite difference code and it is envisaged that other nonNewtonian flows will be studied using this methodology in the future including Maxwell upper convected fluids (Vajravelu,2012) and couple stress fluids (Beg et al., 2012).

\section{Acknowledgements}

The authors appreciate the constructive comments of the reviewers, which led to definite improvement in the paper. The authors are thankful to the management of Madanapalle Institute of Technology \& Science, Madanapalle for providing research facilities in the campus.

\section{NOMENCLATURE}

$C_{f} \quad$ skin friction coefficient

$f \quad$ non-dimensional steam function

$g \quad$ acceleration due to gravity

$k \quad$ thermal conductivity of Jeffery fluid

$c_{p} \quad$ specific heat capacity

$G r \quad$ Grashof (free convection) number

$\mathrm{Nu} \quad$ local Nusselt number

$\mathrm{Pr} \quad$ Prandtl number

De Deborah number

$T \quad$ temperature of the Jeffery fluid

$S \quad$ suction (wall transpiration) parameter

$S \quad$ Cauchy stress tensor

$u, v \quad$ non-dimensional velocity components along the $\mathrm{x}-$ and $\mathrm{y}-$ directions, respectively

$x \quad$ stream wise coordinate

$y \quad$ transverse coordinate

\section{Greek symbols}

$\alpha \quad$ thermal diffusivity

$\beta \quad$ coefficient of thermal expansion

$\lambda \quad$ ratio of relaxation to retardation times

$\lambda_{1} \quad$ retardation time

$\Phi \quad$ azimuthal coordinate

$\eta \quad$ dimensionless radial coordinate

$\mu \quad$ dynamic viscosity

$v \quad$ kinematic viscosity

$\theta \quad$ non-dimensional temperature

$\rho \quad$ density of Jeffery fluid

$\xi \quad$ dimensionless tangential coordinate

$\psi \quad$ dimensionless stream function

\section{Subscripts}

$W$

$\infty$

$T$

$u, v$

$x$

$y$ surface conditions on cylinder (wall) free stream conditions

temperature non-dimensional velocity components along the $\mathrm{x}$ - and $\mathrm{y}-$ directions, respectively stream wise coordinate transverse coordinate 


\section{REFERENCES}

Anwar, I., Amin, N. and Pop, I., 2008, "Mixed Convection Boundary Layer flow of a Viscoelastic fluid over A Horizontal Circular Cylinder," Int. J. Non-Linear Mechanics, 43, 814-821.

https://doi.org/10.1016/j.ijnonlinmec.2008.04.008

Amanulla, C.H., Nagendra, N., Surya Narayana Reddy, M., Subba Rao, A., and Anwar Bég, O., 2017, "Mathematical Study of Non-Newtonian Nanofluid Transport Phenomena from an Isothermal Sphere," Frontiers in Heat and Mass Transfer, 8, 29.

http://dx.doi.org/10.5098/hmt.8.29

Baaijens, F.P.T., S. H. A. Selen, H. P. W. Baaijens, G. W. M. Peters, H. E. H. Meijer, 1997, "Viscoelastic Flow Past A Confined Cylinder of A Low Density Polyethylene Melt," J. Non-Newtonian Fluid Mech. 68, 173-203.

https://doi.org/10.1016/S0377-0257(96)01519-4

Bég, O. Anwar and O.D. Makinde, 2011, "Viscoelastic Flow and Species Transfer in a Darcian High-Permeability Channel," $J$. Petroleum Science and Engineering, 76, 93-99.

https://doi.org/10.1016/i.petrol.2011.01.008

Bég, O. Anwar, Gorla RSR, Prasad VR, Vasu, B., 2011, "Computational Study of Mixed Thermal Convection Nanofluid Flow in A Porous Medium," 12th UK National Heat Transfer Conference, 30th August- 1st September, University of Leeds, UK.

Bég, O. Anwar, H.S. Takhar, G. Nath and M. Kumari, 2001, "Computational Fluid Dynamics Modeling of Buoyancy-Induced Viscoelastic Flow in A Porous Medium with Magnetic Field Effects," Int. J. Applied Mechanics And Engineering, 6, 187-210.

http://e-space.mmu.ac.uk/id/eprint/76539

Bég, O. Anwar, S.K. Ghosh, S. Ahmed and T. A. Bég, 2012, "Mathematical Modelling of Oscillatory Magneto-Convection of A Couple Stress Bio-fluid in an Inclined Rotating Channel," J. Mechanics in Medicine and Biology, 12, 1250050.1 - 1250050.35.

https://doi.org/10.1142/S0219519411004654

Bird, R.B., Armstrong, R.C. and Hassager, O., 1977, "Dynamics of Polymeric Liquids," 1, Fluid Mechanics, Wiley, New York.

Chang, C.L. 2006, "Buoyancy and Wall Conduction Effects on Forced Convection of Micropolar fluid flow Along a Vertical Slender Hollow Circular Cylinder", Int. J. Heat Mass Transfer, 49, 4932-4942.

https://doi.org/10.1016/j.ijheatmasstransfer.2006.05.037

Chen S.S. and Leonard R.1972, "The Axisymmetrical Boundary Layer for a Power-Law Non-Newtonian Fluid on a Slender Cylinder," Chem. Eng. J., 3, 88-92.

https://doi.org/10.1016/0300-9467(72)85009-3

Eswara, A.T. and Nath G. 1992, "Unsteady Forced Convection Laminar Boundary Layer Flow over a Moving Longitudinal Cylinder," Acta Mech., 93, 13-28.

https://doi.org/10.1007/BF01182570

Hayat, T., A. Alsaedi, S.A. Shehzad, 2012, "Three Dimensional Flow of Jeffery Fluid with Convective Surface Boundary Conditions," Int. J. Heat and Mass Transfer, 55, 3971-3976.

https://doi.org/10.1016/j.ijheatmasstransfer.2012.03.027

Hayat.T, S.A. Shehzad, M. Qasim and S.Obaidat, 2012, "Radiative Flow of Jeffery Fluid in a Porous Medium with Power Law Heat Flux and Heat Source," Nuclear Engineering and Design, 243, 15-19.

https://doi.org/10.1016/j.nucengdes.2011.11.005
Hossain, M.A. and R.S.R. Gorla, 2009, "Natural Convection Flow of Non-Newtonian Power-Law Fluid From a Slotted Vertical Isothermal Surface," Int. J. Numerical Methods Heat Fluid Flow, 19, 835-846. https://doi.org/10.1108/09615530910984109

Javed, T., Ali, N., Abbas, Z. and Sajid, M. 2013, "Flow of an EyringPowell Non-Newtonian Fluid over a Stretching Sheet," Chem. Eng. Comm., 200, 327-336. http://dx.doi.org/10.1080/00986445.2012.703151

Kasim, A.R.M., Mohammad N.F. and S. Shafie, 2011, "Effect of Heat Generation on Free Convection Boundary Layer Flow of a Viscoelastic Fluid Past A Horizontal Circular Cylinder With Constant Surface Heat Flux," 5th Int. Conf. Research and Education in Mathematics (ICREM5), Bandung, Indonesia, 22-24 October 2011.

Keller, H.B. 1970, "A new Difference Method for Parabolic Problems," In: J Bramble (ed.) Numerical Methods for Partial Differential Equations. New York: Academic Press.

Kothandapani, M., Srinivas, S. 2008, "Peristaltic Transport of a Jeffery Fluid under the Effect of Magnetic Field in an Asymmetric Channel," Int. J. Nonlinear Mech., 43, 915-924.

https://doi.org/10.1016/j.ijnonlinmec.2008.06.009

Lin, F. N. and Chern, S. Y. 1979, "Laminar Boundary-Layer Flow of Non-Newtonian Fluid," Int. J. Heat Mass Transfer, 22, 1323-1329. https://doi.org/10.1016/0017-9310(79)90194-7

Middleman, S.1977, “Fundamentals of Polymer Processing," Mc Graw Hill, USA.

Nadeem, S., Akbar, N. S., 2009, "Peristaltic Flow of A Jeffery Fluid with Variable Viscosity in an Asymmetric Channel," Z. Naturforsch A. 64a, 713-722.

https://doi.org/10.1515/zna-2009-1107

Nadeem, S., S. Zaheer, and T. Fang, 2011, "Effects of Thermal Radiation on The Boundary Layer flow of a Jeffrey fluid over An Exponentially Stretching Surface," Numerical Algorithms, 57, 187-205. https://doi.org/10.1007/s11075-010-9423-8

Otsuki, Y, Kajiwara T and Funatsu K, 1999, "Numerical Simulations of Annular Extrudate Swell Using Various Types Of Viscoelastic Models," Polymer Engineering \& Science, 39, 1969-1981.

https://doi.org/10.1002/pen.11590

Pop, I., Kumari, M. and Nath, G.1990, "Non-Newtonian Boundary Layers on a Moving Cylinder," Int. J. Eng. Sci., 28, 303-312.

https://doi.org/10.1016/0020-7225(90)90103-P

Prasad V. R., Subba Rao. A, and O. Anwar Beg, 2014, "Computational analysis of Viscous Dissipation and Joule- Heating effects on nonDarcy MHD natural convection flow from a Horizontal Cylinder in Porous Media with Internal Heat Generation," Theoretical and Applied Mechanics.41, .37-70.

https://doi.org/10.2298/TAM1401037P

Prasad, V.R., A. Subba Rao, N. Bhaskar Reddy, B. Vasu, O. Anwar Bég, 2012, "Modelling Laminar Transport Phenomena in a Casson Rheological Fluid from a Horizontal Circular Cylinder With Partial Slip," Proc IMechE- Part E: J. Process Mechanical Engineering.4, 309-326.

https://doi.org/10.1177/0954408912466350 
Ramachandra Prasad V., A. Subba Rao and O. Anwar Beg, 2013, "Flow and Heat Transfer of non-Newtonian Casson fluid from an Isothermal Sphere with Partial Slip in a non-Darcy Porous Medium," Theoretical and Applied Mechanics, 40, 469-510; https://doi.org/10.2298/TAM1303465P

Rehman, M.A. and Nadeem, S, 2012, "Mixed Convection Heat Transfer in Micropolar Nanofluid over a Vertical Slender Cylinder," Chin. Phys. Lett., 29, 124701.

https://doi.org/10.1088/0256-307X/29/12/124701

Rotte, J. W. and Beek, W.J.1969, "Some Models for the Calculation of Heat Transfer Coefficients To Moving Continuous Cylinders," Chem. Eng. Sci., 24, 705-716.

https://doi.org/10.1016/0009-2509(69)80063-1

Roy, S.C., 1971,"Withdrawal of Cylinders from non-Newtonian fluids," Can. J. Chem. Eng. 49, 583-589.

https://doi.org/10.1002/cjce.5450490506

Saasen, A. and Hassager, O., 1991, "Gravity waves and RayleighTaylor instability on a Jeffrey-fluid," Rheologica Acta, 30, 301-306. https://doi.org/10.1007/BF00404190

Sakiadis, B.C. 1961, "Boundary Behavior on Continuous Solid Surfaces, III the Boundary Layer on a Continuous Cylindrical Surface," AIChE. J., 7: 467-472.

https://doi.org/10.1002/aic.690070325

R. Srinivasa Raju, G. Jithender Reddy, J. Anand Rao, M.M. Rashidi, Rama Subba Reddy Gorla, 2016, "Analytical and Numerical Study of Unsteady MHD Free Convection Flow over an Exponentially Moving Vertical Plate With Heat Absorption," International Journal of Thermal Sciences, 107, $303-315$.

https://doi.org/10.1016/j.ijthermalsci.2016.04.005

Subba Rao. A, Nagendra N, Ramachandra Prasad.V, 2015, "Heat Transfer in a Non-Newtonian Jeffery's Fluid over a Non-Isothermal Wedge," Procedia Engineering, 127,775-782.

https://doi.org/10.1016/j.proeng.2015.11.412

Subba Rao. A, Ramachandra Prasad. V, Nagendra. N, Bhaskar Reddy. $\mathrm{N}$ and Anwar Beg.O, 2016, "Non-Similar Computational Solution for Boundary Layer Flows of Non-Newtonian Fluid from an Inclined Plate with Thermal Slip," Journal of Applied Fluid Mechanics, 2, 795-807.

Subba Rao. A., Prasad V.R, Bhaskar Reddy N. and Anwar Beg O. 2015, "Modelling Laminar Transport Phenomena in A Casson Rheological Fluid from an Isothermal Sphere with Partial Slip," Thermal Science, 19, 1507-1519.

Subba Rao. A, Prasad V.R, Bhaskar Reddy N. and Anwar Beg O. 2015, "Modelling Laminar Transport Phenomena in a Casson rheological fluid from a Semi-Infinite Vertical Plate with Partial Slip," Heat Transfer-Asian Research,44,272-291.

Subba Rao. A, Prasad. V. R., Harshavalli. K. and Anwar Beg. O.2016, "Thermal Radiation Effects on Non- Newtonian Fluid in a Variable Porosity Regime with Partial Slip," J. Porous Media, 19, 313-329. https://doi.org/10.1615/JPorMedia.v19.i4.30

Rao, S.A., Amanulla, C.H., Nagendra, N., Bég, O. A., Kadir, A., 2017, "Hydromagnetic flow and Heat Transfer in a Williamson NonNewtonian fluid from a Horizontal Circular Cylinder with Newtonian Heating," Int. J. Appl. Comput. Math., 1-21.

http://dx.doi.org/10.1007/s40819-017-0304-x

Rao, S.A., Amanulla, C.H., Nagendra, N., Surya Narayana Reddy, M., and Bég, O.A., 2017, "Computational Analysis of Non-Newtonian Boundary Layer Flow of Nanofluid Past a Vertical Plate with Partial Slip," Modelling, Measurement and Control B, 86(1), 271-295. http://usir.salford.ac.uk/42310/

Tripathi, D., Bég, O. Anwar and Curiel-Sosa. J 2014, "Homotopy SemiNumerical Simulation of Peristaltic Flow of Generalized Oldroyd-B Fluids with Slip Effects," Computer Methods in Biomechanics Biomedical Engineering, 17, 433-442.

http://dx.doi.org/10.1080/10255842.2012.688109

Vajravelu, K., K.V. Prasad and C-O. Ng, 2012, "Unsteady Flow and Heat Transfer in a Thin Film of Ostwald-De Waele Liquid over a Stretching Surface," Commun. Nonlinear Sci. Numer. Simulation, 17, 4163-4173.

https://doi.org/10.1016/j.cnsns.2012.01.027

Vajravelu, K., K.V. Prasad, A. Sujatha and C-O. Ng, 2012, "MHD Flow and Mass Transfer of Chemically Reactive Upper Convected Maxwell fluid Past Porous Surface," Applied Mathematics and Mechanics, 33, 899-910.

https://doi.org/10.1007/s10483-012-1593-8

Vrentas, J.S., Jarzebski, C.M. and J. L. Duda, 1975, "A Deborah Number for Diffusion In Polymer-Solvent Systems," AIChE J., 21, 894-901.

https://doi.org/10.1002/aic.690210510

Zueco, J., O. Anwar Bég, H. S. Takhar and G. Nath, 2011, "Network Simulation of Laminar Convective Heat and Mass Transfer over A Vertical Slender Cylinder with Uniform Surface Heat and Mass Flux," J. Applied Fluid Mechanics, 4, 13-23.

Zueco, J., O. Anwar Bég, Tasveer A. Bég and H.S. Takhar, 2009, "Numerical Study of Chemically-Reactive Buoyancy-Driven Heat and Mass Transfer across a Horizontal Cylinder in A High-Porosity NonDarcian Regime," J. Porous Media, 12, 519-535.

https://doi.org/10.1615/JPorMedia.v12.16.30 